\title{
The multinational second Diabetes, Attitudes, Wishes and Needs study: results of the French
}

\section{survey}

\author{
Gérard Reach ${ }^{1,2}$ \\ Silla M Consoli ${ }^{3,4}$ \\ Serge Halimi ${ }^{5}$ \\ Claude Colas ${ }^{6}$ \\ Martine Duclos ${ }^{7-9}$ \\ Pierre Fontaine ${ }^{10}$ \\ Caroline Martineau'" \\ Carole Avril ${ }^{12}$ \\ Catherine Tourette-Turgis ${ }^{13}$ \\ Sylvie Pucheu ${ }^{4}$ \\ Olivier Brunet ${ }^{14}$ \\ 'Department of Endocrinology, Diabetes and \\ Metabolic Diseases, Avicenne Hospital, Bobigny, \\ 'EA 34I2, CRNH-IDF, Paris 13 University, \\ Sorbonne Paris Cité, Bobigny, ${ }^{3}$ Paris Descartes \\ University, Paris Sorbonne Cité, Faculty of \\ Medicine, Paris, ${ }^{4}$ University Service of Adult \\ and Elderly Psychiatry, European Georges- \\ Pompidou Hospital, Paris, ${ }^{5}$ Department of \\ Diabetology, Endocrinology and Nutrition, \\ Joseph Fourier University and CHU Grenoble, \\ Grenoble, ${ }^{6}$ Department of Diabetology, \\ Hôtel-Dieu Hospital, Paris, ${ }^{7}$ Department of \\ Sport Medicine and Functional Explorations, \\ Montpied Hospital, Clermont-Ferrand, \\ ${ }^{8}$ Department of Human Nutrition, Centre \\ de Recherche en Nutrition Humaine, Institut \\ National de la Recherche Agronomique, \\ Clermont-Ferrand, ' Department of Human \\ Nutrition, University of Auvergne, Clermont- \\ Ferrand, ${ }^{10}$ Department of Endocrinology, \\ Diabetes and Metabolic Diseases, Huriez \\ Hospital, Lille 2 University, Lille, "'Department \\ of Dietetics, Larrey Hospital, CHU \\ Toulouse, ${ }^{12}$ French Diabetics Federation, \\ Paris, ${ }^{13}$ UPMC-Sorbonne Universités, Paris, \\ ${ }^{14}$ General Practitioner, Seraincourt, France
}

Correspondence: Gérard Reach

Department of Endocrinology, Diabetes, and Metabolic Diseases, Avicenne Hospital APHP, 125 rue de Stalingrad, 93000 Bobigny, France Tel +33 | 48955158

Fax +33 | 48955560

Email gerard.reach@avc.aphp.fr
This article was published in the following Dove Press journal:

Patient Preference and Adherence

12 February 2015

Number of times this article has been viewed

Aim: The second Diabetes, Attitudes, Wishes and Needs (DAWN2' ${ }^{\mathrm{TM}}$ ) multinational cross-sectional study was aimed at generating insights to facilitate innovative efforts by people with diabetes (PWD), family members (FMs), and health care professionals (HCPs) to improve self-management and psychosocial support in diabetes. Here, the French data from the DAWN2 ${ }^{\mathrm{TM}}$ study are described.

Methods: In France, 500 PWD ( 80 with type 1 diabetes [T1] and 420 with type 2 diabetes [T2]), $120 \mathrm{FMs}$, and $288 \mathrm{HCPs}$ were recruited. The questionnaires assessed the impact of diabetes on quality of life and mood, self-management, attitudes/beliefs, and care/support.

Results: Diabetes negatively impacted the emotional well-being of 59\% of people with T1 versus $45 \%$ of people with T2 $(P<0.05)$ and about half of FMs. A high level of distress was felt by about half of PWD and FMs. About half of HCPs reported assessing depression in their patients. Sixty-two percent of FMs considered managing diabetes to be a burden. Hypoglycemia was a source of concern for $64 \%$ of people with T1 and 73\% of FMs of insulin users. About two-thirds of non-insulin-medicated people with T2 agreed to start insulin if prescribed, while half of HCPs preferred to delay insulin initiation. A discrepancy between HCPs' perceptions of their interactions with their patients and PWD's recollection of these interactions with regard to patients' personal needs and distress was also observed.

Conclusion: While distress remains under-assessed by HCPs, the negative impact of diabetes on the lives of PWD and FMs clearly induces distress on both groups. These findings provide new understanding of barriers precluding optimal management of diabetes. Developing strategies to overcome these barriers is now warranted.

Keywords: health care provision, household study, psychosocial, quality of life

\section{Introduction}

A decade ago, the Diabetes, Attitudes, Wishes and Needs (DAWN) study highlighted the importance of paying close attention to both the medical and psychosocial needs of people with diabetes (PWD). Indeed, the DAWN study reported a critical discrepancy between the psychosocial and educational support needs of PWD, and the care and support available. ${ }^{1}$ Furthermore, effective collaboration among diabetes care providers was defined as a key factor for improving diabetes outcomes. ${ }^{2}$

During the past decade, significant advancements have been made. Indeed, the International Diabetes Federation (IDF) Global Guidelines for type 2 diabetes underlines the importance of regularly assessing the psychosocial well-being (eg, depression, anxiety, diabetes-specific distress, and denial of the condition) and requiring training of health care professionals (HCPs) to recognize psychological problems. ${ }^{3}$ Along with these recommendations, the acute care model has evolved 
toward a more patient-centered, bio-psychosocial care model, encompassing patients' engagement in care, shared decision making, and consideration of patients' preferences and environment. ${ }^{4}$ Incidentally, this represents the basis for the new patient-centered guidelines issued by the American Diabetes Association and the European Association for the Study of Diabetes, ${ }^{5}$ and, in France, by the Haute Autorite de Santé. ${ }^{6}$ Despite this progress, there are still far too many PWD who are not getting the care and support they need, which could enable them to achieve optimal health and well-being. Therefore, access to patient-centered chronic care, education, and support needed to be improved.

In response, the second DAWN (DAWN2 ${ }^{\mathrm{TM}}$ ) study was designed. This multinational study was aimed at generating insights that can facilitate innovative efforts by PWD, their family members (FMs), and HCPs to improve self-management and psychosocial support in diabetes. Consequently, the DAWN2 ${ }^{\mathrm{TM}}$ study constitutes the first major survey with the aim of gathering the opinions of the FMs of PWD. ${ }^{7}$ The primary objective of this study was to assess and explore potential drivers of active and successful diabetes management among PWD, their FMs, and HCPs. In this article, we examine French data from the DAWN2 ${ }^{\mathrm{TM}}$ study.

\section{Materials and methods Study design}

DAWN2 ${ }^{\mathrm{TM}}$ is a cross-sectional, international, interdisciplinary, multi-stakeholder study conducted in 17 countries from four continents between March and August 2012 and whose methodology has been published elsewhere. ${ }^{7}$ In France, the DAWN2 ${ }^{\text {TM }}$ study was compliant with the French data protection authority (Commission Nationale de l'Informatique et des Libertés [CNIL]), ie, CNIL's registry requirements, was conducted in accordance with the relevant guidelines relating to the conduct of noninterventional studies, and used the International Chamber of Commerce/European Society for Opinion and Marketing Research, the Council of American Survey Research Organizations, and Good Pharmacoepidemiology Practices guidelines as a minimum standard. Data collection was anonymous.

\section{Participants}

In France, the study sample was comprised of 500 PWD who were diagnosed for no less than 12 months, $120 \mathrm{FMs}$, and 288 HCPs. All 908 participants were recruited by a hybrid online and telephone methodology. To ensure a study population as representative as possible of the wider diabetes community, the methodology of PWD recruitment was established by stratification on quotas of age, sex, socioeconomics, education level, and other demographic information, which were based on the IDF database. According to quota sampling, the PWD sample, all 18 years or older, were comprised of 80 type 1 PWD (T1) and 420 type 2 PWD (T2), including 100 nonmedicated T2 (T2 nonmedicated) under diet and exercise regimen only, 170 non-insulin medicated T2 (T2 non-insulin medicated), and 150 insulinmedicated T2 (T2 insulin-medicated). T1 had started insulin treatment at diagnosis, which was established before the age of 30 years, and were still under insulin treatment at the time of the study, while T2 did not start insulin treatment at diagnosis, which was established at or after the age of 30 years. Excluded PWD were those under 18 years old, those whose diabetes had been diagnosed for less than 12 months, and/or those whose diabetes was related to pregnancy.

The FMs sample comprised FMs of insulin-medicated and non-insulin medicated PWD (FMs insulin-medicated and FMs non-insulin medicated, respectively). FMs were not diagnosed with diabetes, were living in the same household with a PWD, and were involved in his/her care. Only two FMs were linked to the interviewed PWD; the other 118 FMs were not related to the interviewed PWD.

The HCP sample comprised 120 general practitioners (GPs); 80 specialists (Sps), mainly diabetologists, endocrinologists, and internists; and 48 nurses and 40 dietitians (NDs). All HCPs had been in practice for over a year. GPs and NDs personally treated at least five PWD per month, and Sps at least 50 PWD per month. GPs were primary care physicians and internal medicine physicians who initiated oral medication, while Sps were endocrinologists/diabetologists or GPs with subspecialty in diabetes who prescribed insulin among other diabetes medications. HCPs were identified from online panels and databases.

\section{Assessment}

Three separate survey questionnaires, one for each of the three diabetes stakeholder groups, were designed to permit comparison across respondent types where possible. All three questionnaires were web-based and were completed predominantly online, or via a telephone interview. These were conducted by professionally trained interviewers, employed by survey research companies based in France, themselves employed by Harris Interactive Inc. (Rochester, USA), the company globally responsible for undertaking the survey. The questionnaires covered a wide range of topics including the impact of diabetes on health and quality of life (QoL), care and support, self-management, involvement/role of FMs, as well as beliefs and attitudes. To explore these topics, the 
questionnaires incorporated items from the original DAWN study; new questions developed by a multi-disciplinary, multinational team; as well as standardized instruments including World Health Organization Quality of Life-BREF (WHO QOL-BREF), ${ }^{8}$ EuroQoL-5D, ${ }^{9}$ World Health Organization 5-item Well-Being Index (WHO-5), ${ }^{10}$ the Problem Areas in Diabetes 5 Short Form (PAID-5-SF), ${ }^{11,12}$ the Problem Areas in Diabetes Distress of Family Members (PAID-5DFM), the Health Care Climate Questionnaire (HCC), ${ }^{13}$ and the Summary of Diabetes Self-Care Activities Measure (SDSCA). ${ }^{14}$ In addition, questions integrated in the questionnaires were developed with inspiration from or were adapted or modified from existing validated instruments including the Diabetes Empowerment Scale-Short Form (DES-SF), ${ }^{15}$ Helpfulness Active Patient Involvement-DAWN Short Form (HAPI-DSF), ${ }^{16}$ Patient Assessment of Chronic Illness Care (PACIC), ${ }^{17,18}$ Diabetes Family Behavior Checklist, ${ }^{19}$ and DAWN Impact of Diabetes Profile (DIDP). ${ }^{20}$ Open-ended questions were also used to capture anecdotal information.

\section{Statistical methods}

Descriptive data, presented as means (quantitative variables) or percentages (categorical variables), were unweighted data for within country comparisons, and cluster-adjusted data for comparisons between France and worldwide data. Adjustment for clustering within countries was performed using multilevel regression models with an unstructured correlation-type matrix; to allow for generalizability, data for each country were also weighted on age, sex, region, and education according to population proportions, which were provided by local survey advisory groups, and coming from publicly available epidemiological data. Differences among respondent groups were tested using the chi-squared test, Kendall correlation coefficient, Mann-Whitney test, and Kruskal-Wallis test. Statistical significance was set at $P<0.05$. Comparisons presented in the figures were made by population type: PWD, FMs or HCPs.

\section{Results PWD, FM, and HCP characteristics}

PWD characteristics are shown in Table 1. For FMs, the mean age was 35.6 years, $43 \%$ of whom were men and $58 \%$ women. Non-insulin medicated PWD were more often a relative of FMs in the non-insulin medicated group compared with the insulin-medicated group (52\% versus $27 \%$, $P<0.05$ ). Also, $35 \%$ and $26 \%$ of FMs were a spouse/partner of the insulin-medicated and non-insulin medicated PWD, respectively. Among HCPs, GPs and Sps were mostly males ( $86 \%$ and $53 \%$, respectively), while NDs were primarily females $(89 \%)$. Eighty-seven percent of GPs were private practice-/office-based while $46 \%$ of Sps and $49 \%$ of NDs were hospital-based.

\section{Diabetes profile}

As shown in Table 1, at least one in four PWD had both physical and emotional associated disorders. Sleeping problems (ranging from $36 \%$ to $46 \%$ ) and depression (ranging from $20 \%$ to $28 \%$ ) were the most commonly mentioned disorders according to PWD respondents.

Among all T2, 60\% used diet and exercise, 58\% used pills or tablets to manage their diabetes, and $36 \%$ were on insulin, whereas $46 \%$ of $\mathrm{T} 1$ reported using diet and exercise and

Table I Patients characteristics

\begin{tabular}{|c|c|c|c|c|c|c|}
\hline & \multirow{2}{*}{$\begin{array}{l}\mathrm{TI}+\mathrm{T} 2 \\
\mathrm{n}=500\end{array}$} & \multirow{2}{*}{$\begin{array}{l}\text { TI } \\
\mathrm{n}=\mathbf{8 0}\end{array}$} & \multicolumn{4}{|l|}{ T2 } \\
\hline & & & $T 2 n=420$ & $\begin{array}{l}\text { T2 nonmedicated } \\
n=100\end{array}$ & $\begin{array}{l}\text { T2 non-insulin } \\
\text { medicated } n=170\end{array}$ & $\begin{array}{l}\text { T2 insulin-medicated } \\
n=\mid 50\end{array}$ \\
\hline \multicolumn{7}{|l|}{ Sex } \\
\hline Male & $55 \%$ & $46 \%$ & $56 \%$ & $51 \%$ & $58 \%$ & $57 \%$ \\
\hline \multicolumn{7}{|l|}{ Age (years) } \\
\hline $18-39$ & $10 \%$ & $38 \%$ & $5 \%$ & $16 \%$ & $1 \%$ & $3 \%$ \\
\hline $40-59$ & $48 \%$ & $53 \%$ & $47 \%$ & $60 \%$ & $39 \%$ & $48 \%$ \\
\hline$\geq 60$ & $42 \%$ & $10 \%$ & $48 \%$ & $24 \%$ & $61 \%$ & $49 \%$ \\
\hline Mean BMI $\left(\mathrm{kg} / \mathrm{m}^{2}\right)$ & 28.9 & 24.6 & 29.7 & 28.5 & 31.2 & 28.7 \\
\hline \multicolumn{7}{|l|}{ Associated disorders } \\
\hline Physical & $21 \%$ & $17 \%$ & $21 \%$ & $18 \%$ & $24 \%$ & $21 \%$ \\
\hline Emotional & $18 \%$ & $18 \%$ & $19 \%$ & $22 \%$ & $19 \%$ & $15 \%$ \\
\hline Both physical and emotional & $30 \%$ & $34 \%$ & $30 \%$ & $31 \%$ & $25 \%$ & $35 \%$ \\
\hline No other disorder & $30 \%$ & $31 \%$ & $30 \%$ & $29 \%$ & $32 \%$ & $29 \%$ \\
\hline
\end{tabular}

Notes: Physical associated disorders included stroke, open wound on the foot that did not heal without medical care, entire or partial foot/leg amputation, kidneys not working properly, eyesight damage, nerve damage, problems with sexual functioning, heart disease, and heart attack. Emotional associated disorders included depression and sleeping problems. Both physical disorders and emotional disorders concerns patients displaying only disorders from the corresponding categories. Abbreviations: BMI, body mass index; TI, type I diabetes; T2, type 2 diabetes. 
$15 \%$ took pills or tablets. Ten percent of PWD treated their diabetes with alternative medicine (ie, herbal, acupuncture, or homeopathy) and 7\% with other injectable medications.

GPs and Sps estimated that $45 \%$ and $31 \%$ of their patients had glycated hemoglobin levels $<7 \%$, respectively.

\section{QoL}

The impact of diabetes on several aspects of the QoL of PWD and FMs, assessed by DIDP, is presented in Figure 1. Diabetes had a negative impact on the QoL of PWD, mainly of T1. Thirty-seven percent of T1 and $41 \%$ of T2 presented with a WHO-5 score suggesting depressive mood and 14\% of T1 and T2 with likely depression. The main domains of altered QoL in PWD were physical health, emotional wellbeing, and leisure activities. The majority of T1 (78\%) and T2 (67\%) reported that diabetes had "slightly to very negative impact" on their physical health. The negative impact of diabetes on emotional well-being was greater in T1 than in T2 (59\% versus $45 \%$, respectively, $P<0.05$ ). Leisure activities were also more affected in T1 than in T2 (58\% versus $42 \%$, respectively, $P<0.05)$. Consistently, $44 \%$ of PWD felt a high level of distress as measured by PAID-5 scale.

DIDP showed that diabetes also had an impact on the QoL of FMs. Half of FMs reported a negative impact of diabetes on their own emotional well-being (Figure 1). Similarly to what PWD reported, diabetes affected leisure activities in $35 \%$ and $15 \%$ of FMs insulin-medicated and FMs noninsulin medicated, respectively. Data from the PAID-5-DFM scale indicated that worrying about the future and possible complications was a "serious or somewhat serious" problem for 33\% and 53\% of FMs non-insulin medicated and FMs insulin medicated, respectively $(P<0.05)$. Consistent with this data, 59\% of FMs insulin-medicated and $65 \%$ of FMs non-insulin medicated considered that managing the diabetes of the person they live with was a moderate to very large burden. However, 47\% of FMs reported through DIDP that helping a PWD had a positive impact on at least one aspect of their life. Indeed, respectively, 64\% and 63\% of FMs insulin medicated and FMs non-insulin medicated agreed that they had learned to take better care of their own health. Moreover, 70\% and 59\% of FMs insulin-medicated and FMs non-insulin medicated, respectively, declared that they had found good ways to help the person they live with by taking care of his/her diabetes.

\section{Attitude and beliefs}

Overall, attitudes about diabetes were largely shared by PWD and FMs: $86 \%$ of T1 and $89 \%$ of T2 agreed that complications can be avoided if the condition is carefully managed, and, respectively, $89 \%$ and $81 \%$ of FM insulin-medicated and FM non-insulin medicated had the same perception about the relationship between diabetes management and complications. Across all groups, most PWD were willing to follow their HCP's recommendations. Sixty-six percent of T2 non-insulin medicated and $57 \%$ of $\mathrm{T} 2$ nonmedicated said they were willing to start glucagon-like peptide-1 analogs, and $72 \%$ of T2 noninsulin medicated and $61 \%$ of T 2 nonmedicated said they were willing to start insulin if prescribed. Conversely, GPs and Sps preferred to delay the prescription of insulin

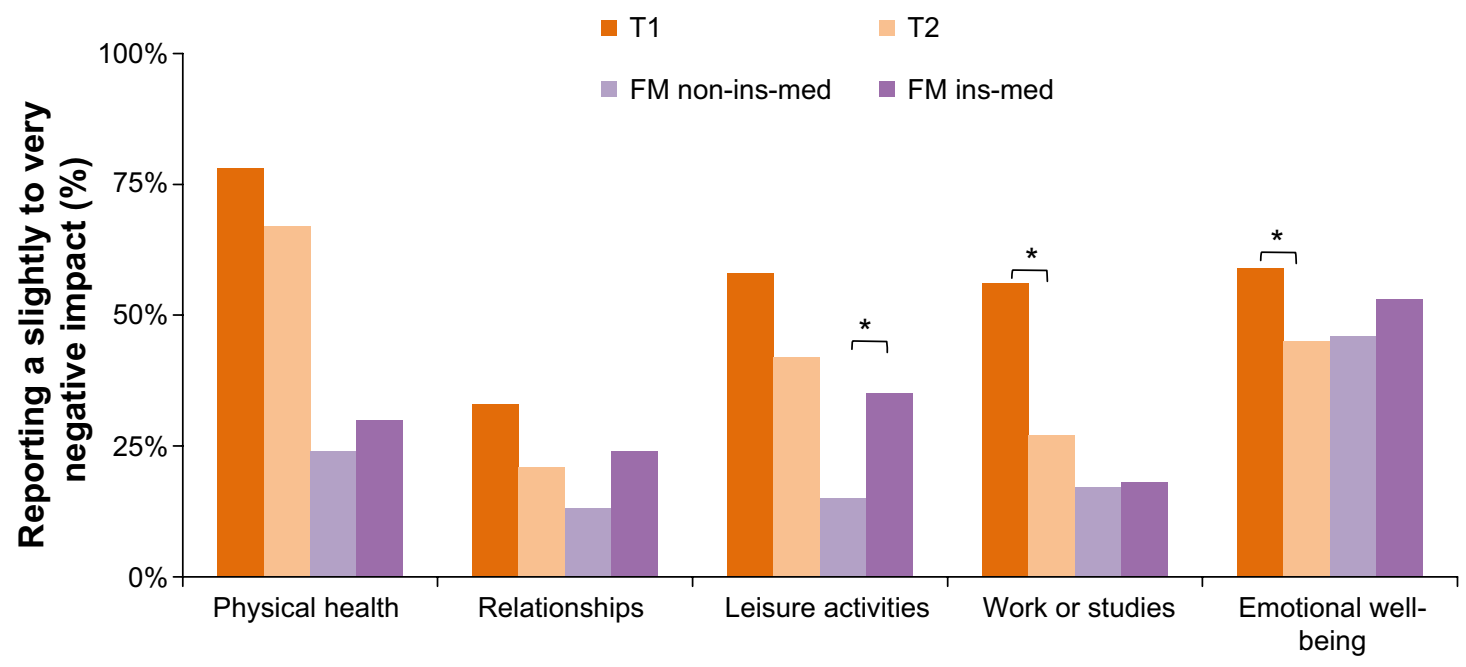

Figure I Impact of diabetes on quality of life.

Notes: The impact of diabetes was assessed by the DAWN Impact of Diabetes Profile questionnaire. *Indicates statistical significance between subgroups, which was set at $P<0.05$.

Abbreviations: DAWN, Diabetes, Attitudes, Wishes and Needs; FM non-ins-med, family member of non-insulin-medicated people with diabetes; FM ins-med, family member of insulin-medicated people with diabetes; TI, type I diabetes; T2, type 2 diabetes. 
(56\% and $41 \%$, respectively) and glucagon-like peptide- 1 analogs (37\% and 15\%, respectively) until it becomes absolutely necessary.

Hypoglycemia was a source of concern not only for T1, but also for FMs insulin-medicated. Accordingly, $64 \%$ of T 1 and $73 \%$ of FMs insulin-medicated worried about the risk of night and day hypoglycemic events. Concurrently, $73 \%$ of $\mathrm{T} 1$ and $42 \%$ of $\mathrm{T} 2$ insulin-medicated experienced symptoms of hypoglycemia several times a month or as often as once a week $(P<0.05)$, with both groups reporting an average of two severe low-blood-sugar episodes in the past 12 months. In T2 non-insulin medicated, $22 \%$ reported symptoms of hypoglycemia at least once a week or several times a month with an average of 0.7 severe low-blood-sugar episodes in the past 12 months. Incidentally, lowering the hypoglycemia risk was one of the improvements in diabetes medication that $73 \%$ of GPs and $85 \%$ of Sps would find most helpful to achieve better patient outcomes.

\section{Active self-management}

DES-SF showed that $\mathrm{T} 2$ were more reluctant than $\mathrm{T} 1$ to ask others for support or to even communicate how others could best support them in managing their diabetes $(74 \%$ versus $58 \%, P<0.05)$. Consistently, T1 were more likely than $\mathrm{T} 2$ to have someone other than a HCP involved in helping them manage their diabetes ( $80 \%$ versus $65 \%, P<0.05$ ). HAPI-DSF showed that $23 \%$ of GPs, $33 \%$ of Sps, and $45 \%$ of NDs agreed that it is very helpful when patients let HCPs know how they can best support them in managing their diabetes.
Concerning adherence to HCP recommendations, SDSCA showed that PWD reported that they took all of their HCP recommended medications on average about 6 days per week, followed a healthy eating plan at least 4 days per week, but exercised less than 4 days per week. As shown in Figure 2, PWD, FMs, and HCPs all agreed that eating healthily, exercising, and maintaining a healthy weight were the key areas where substantial improvement is needed.

\section{Care and support}

Almost all PWD had seen a HCP for their diabetes in the past 12 months. The proportion of T2 visiting a GP was higher compared with T1 ( $87 \%$ versus $69 \%, P<0.05)$. Conversely, the proportion of T1 visiting a Sp was higher compared to T2 ( $74 \%$ versus $43 \%, P<0.05$ ). Half of PWD considered their health care team to be very supportive. However, only onethird reported that their HCP asked if they had been anxious or depressed whereas about half of HCPs reported assessing depression in their patients. The perceptions of these two stakeholder groups was assessed by PACIC and HCC. This disparity between HCPs' perceptions of their interactions with their patients and the patients' recollection of these interactions is further illustrated in Figure 3. About $10 \%$ of PWD reported that their HCPs "always" asked them if they had problems with their medications and subsequent effects, encouraged them to ask questions, and listened to how they would like to do things. On the other hand, on average, approximately $25 \%$ of GPs, one-third of Sps, and more than half of NDs reported to "always" ask these questions.

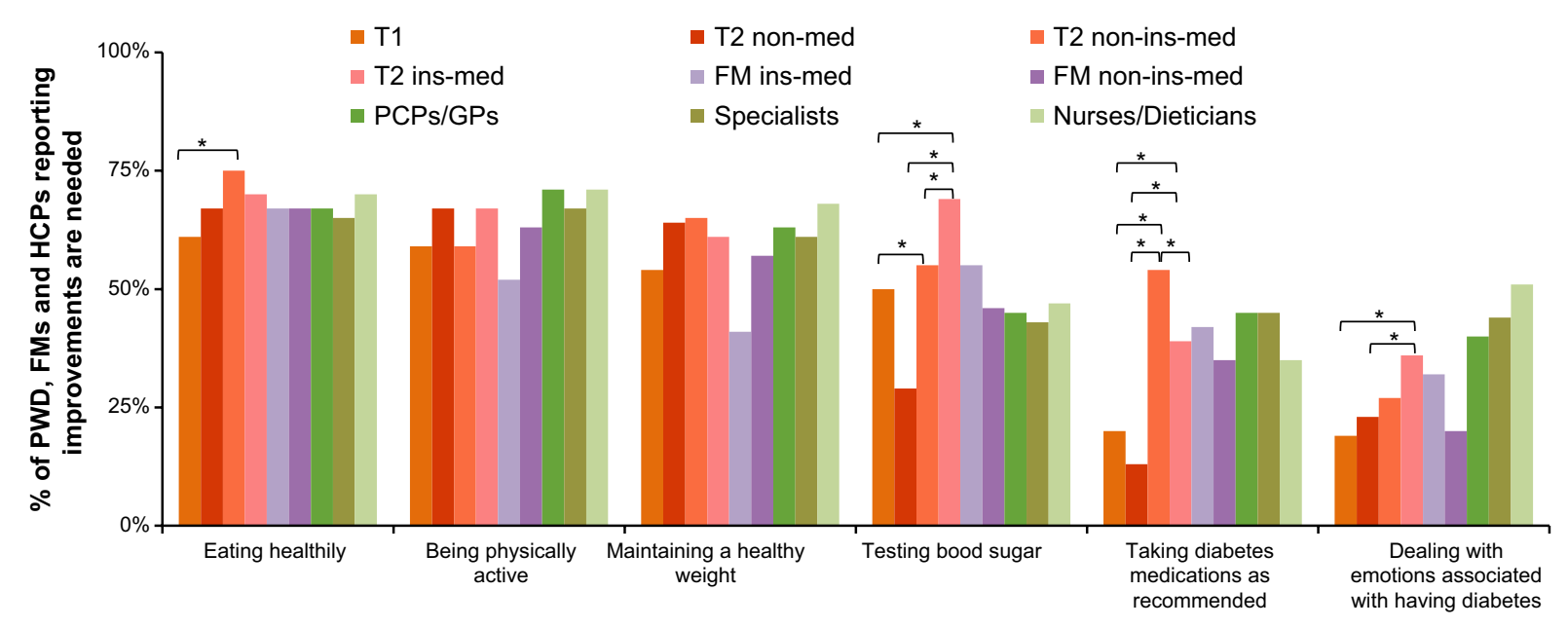

Figure 2 Self-care improvement: PWD's, FMs', and HCPs' perspectives.

Note: *Indicates statistical significance between subgroups, which was set at $P<0.05$. Comparisons were made by population type: PWD, FMs or HCPs.

Abbreviations: FMs, family members; FM ins-med, family members of insulin-medicated PWD; FM non-ins-med, family member of non-insulin-medicated PWD; HCPs, health care professionals; PCPs/GPs, primary care physicians/general practitioners; PWD, people with diabetes; TI, type I diabetes; T2 ins-med, insulin-medicated people with type 2 diabetes; T2 non-ins-med, non-insulin-medicated people with type 2 diabetes; T2 non-med, nonmedicated people with type 2 diabetes. 


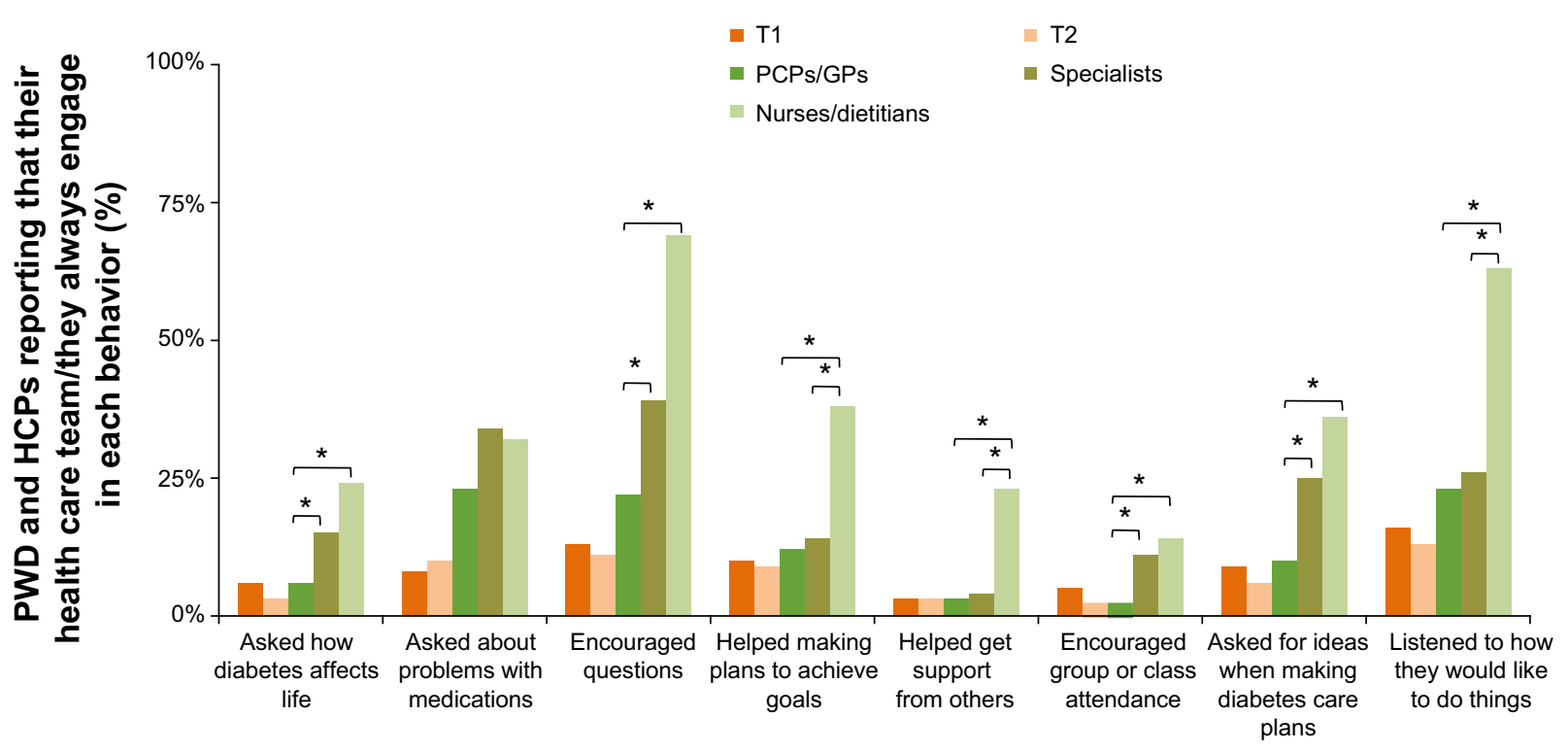

Figure 3 Health team support behaviors: PWD's and HCPs' perspectives.

Notes: The stakeholders' perspective was assessed by the Patient Assessment of Chronic Illness Care and the Health Care Climate Questionnaire questionnaires. *Indicates statistical significance between subgroups, which was set at $P<0.05$. Comparisons were made by population type: PWD on one hand, HCPs on the other hand.

Abbreviations: HCPs, health care professionals; PCPs/GPs, primary care physicians/general practitioners; PWD, people with diabetes; TI, type I diabetes; T2, type 2 diabetes.

\section{Discussion}

The findings from this study of 908 participants underline the impact diabetes has on PWD, and FMs. It also confirms that although depression is a common disorder in PWD, this emotional disorder remains under-assessed by HCPs. Altogether, these results suggest that providing support is necessary, not only for PWD but also for their FMs, to achieve successful management of diabetes.

To have a holistic perspective of the effect of diabetes, its impact on several aspects of the life of the PWD was evaluated in this study. The most negatively impacted aspects of QoL were physical health, emotional well-being, and leisure activities, with physical health and leisure activities being reported as more impacted in France than for the global international set of PWD (65.1\% versus $61.2 \%$, and $40.5 \%$ versus $36.1 \%$, respectively). ${ }^{21}$ This negative impact could be attributed to the fear of hypoglycemia, which was a major concern notably in T1. Hence, this study further indicates that $\mathrm{T} 1$ felt a greater negative effect from diabetic conditions on emotional well-being and leisure activities than did T2. This data is consistent with that of previous studies ${ }^{22}$ which showed that the QoL of PWD was reduced and that the magnitude of negative impact differed between $\mathrm{T} 1$ and $\mathrm{T} 2 .^{23,24}$

In line with the impairment of QoL, approximatively halh of PWD experienced a high level of distress according to PAID-5 scale, in France, versus $41 \%$ for the global international set of PWD. ${ }^{21}$ The WHO-5 scale showed that
$14.0 \%$ of PWD for France versus $14.8 \%$ for the global international set of $\mathrm{PWD}^{21}$ suffered from likely depression in this study, which is consistent with the depression prevalence reported in the literature. ${ }^{25}$ Despite this apparent problem, HCPs seemed reluctant to manage the psychological problems of PWD as only one-third of PWD reported that their HCPs discussed their distress. This point was previously addressed in the original DAWN study, which showed that HCPs felt unable to manage their patients' psychological needs. ${ }^{1}$ Interestingly, when compared with patient reports, a higher proportion of HCPs reported that they "always" ask psychological questions. This apparent misreporting by HCPs may be an effect of denial, in the framework of the effect of denial on HCPs' clinical inertia. ${ }^{26}$ Considering that depression had been associated with poor glycemic control, ${ }^{27}$ complications from diabetes,${ }^{28}$ and increased mortality, ${ }^{29}$ the under-assessment of emotional distress and depression reported in this study suggests that strategies need to be implemented to provide adequate psychological management and support for patients suffering from these problems. Indeed, as previously underlined in the original DAWN study, ${ }^{1}$ HCPs should receive appropriate training to better identify among their patients those suffering from depression and subsequently refer them to psychiatrists for confirmation of the diagnosis.

FMs of PWD reported a negative impact on various life dimensions due to the diabetes of the person they care 
for or live with. Diabetes clearly affected their emotional well-being (49.0\% versus $44.5 \%$ for France versus the global international set of PWD, assessed by DIDP), ${ }^{20}$ which could be related to the concerns that FMs expressed about the future of the person with diabetes or the risk of hypoglycemia as well as the burden they felt from caring for the person with diabetes. These findings are consistent with those of previous studies, showing that diabetes affects both the persons with diabetes and their relatives. ${ }^{30,31}$ This highlights that the psychological problems of FMs should be addressed to support them in the management of the diabetes of the person they care for or live with. An important point of this study was the assessment of the positive impact of diabetes on FMs (45.9\% versus $34.5 \%$ for France versus global, assessed by DIDP). ${ }^{20}$ The majority of FMs found that helping the person with diabetes had a positive impact on at least one aspect of their own life; however, the proportion of FMs reporting a moderate to very large burden in helping the person they live with manage their diabetes radically differs between France, displaying the higher percentage, and the global international set of FMs $(60.0 \%$ versus $33.7 \%$ for France). ${ }^{20}$ This concurrent reporting of negative and positive impacts of diabetes in this study suggests that FMs have to deal with complex feelings about living with a person with diabetes.

Efforts are also required to overcome the resistance to insulin therapy. Indeed, in this study, HCPs preferred to delay the insulin initiation until it was absolutely necessary. By contrast, the vast majority of T2 non-insulin medicated were willing to start insulin if prescribed, possibly because insulin therapy is viewed with a distant future perspective. However, these findings differ from those of previous studies reporting greater insulin-therapy reluctance among PWD. ${ }^{32,33}$ This willingness to start insulin if necessary may be linked to the understanding of most patients and FMs that an appropriate management plan may help in avoiding complications. It alternatively may be linked to a change of appreciation by patients and FMs concerning the real burden of insulin therapy.

This study also points out the discrepancy between HCPs' perceptions of their interactions with patients and the patients' recollection of these interactions. While few PWD felt the need to participate in the medical decisionmaking process, about one-quarter of HCPs reported to have involved their patients in this process. Although we cannot link specific PWD with their own HCP, our results suggest a lack of communication between the patient and the provider in general. This assumption is supported by other studies indicating differences in the concepts and perspectives of PWD and HCPs. ${ }^{34}$ Given that improved patient-provider communication may enhance diabetes self-care and diabetes outcomes, ${ }^{35}$ our study identifies a key driver of successfully managed diabetes.

There are limitations to this study. First, we acknowledge that contributing PWD, and FMs of PWD, may exhibit higher levels of adherence and satisfaction than the population from which they were drawn. Unfortunately, detailed information on PWD who did not participate in the study cannot be provided due to the hybrid recruitment methodology. Likewise, HCPs who participated in the DAWN2 ${ }^{\text {TM }}$ study may declare themselves more attentive to their patients' feelings that the average real-life standard care. This could explain the high discrepancy observed between both stakeholders' interaction recollection. Second, as most FMs and patients were not linked, we do not know whether the attitudes of PWD and their FMs toward diabetes are related to each other. Thirdly, the statistical model of this descriptive study was not powerful enough as confounders were not controlled for, and, as data were unweighted, the results of this study and the subsequent discussion may not be fully representative of the national global population.

\section{Conclusion}

In conclusion, the DAWN2 ${ }^{\mathrm{TM}}$ study has provided new understanding of the barriers precluding the optimal management of diabetes. These barriers notably include the distress and burden felt by the PWD and by FMs. Despite this negative impact of diabetes on emotional status, this study indicates that HCPs experience difficulty in handling the psychological problems of PWD, or find that they do not have adequate resources/training to effectively manage these problems or actively encourage patients to manage their condition. Consequently, innovative strategies must now be developed to overcome these barriers and achieve successful diabetes management with improved outcomes.

\section{Acknowledgments}

We thank ClinSearch (Bagneux, France) who provided medical writing assistance on behalf of Novo Nordisk. This study was supported by Novo Nordisk.

\section{Disclosure}

CA has participated in an advisory board for Novo Nordisk concerning results of the DAWN2 ${ }^{\mathrm{TM}}$ study. OB has received fees for advisory board participation for Novo Nordisk and Boehringer Ingelheim. CC has received fees 
for participation in an advisory board for Abbott, Novo Nordisk, Eli Lilly, Takeda, and Bristol-Myers Squibb. SMC has received fees for participation in an advisory board for Novo Nordisk, and for lectures from AstraZeneca, Bayer, Boehringer Ingelheim, Bristol-Myers Squibb, Eli Lilly, Euthérapie, GlaxoSmithKline, Gilead, Janssen, Lundbeck, MSD, Merck Sharp and Dohme, Novartis, Novo Nordisk, Otsuka Pharmaceutical France, Pfizer, Sanofi, Servier, and Wyeth. MD has received fees for advisory board participation for Novo Nordisk; she has participated in an advisory board, was a speaker, and was responsible for the coordination and moderation of medico-scientific sessions for Novartis. PF has received fees for participation in an advisory board for Novo Nordisk (and for the analysis of the results of the DAWN2 ${ }^{\mathrm{TM}}$ study), Eli Lilly, Sanofi, Merck Sharp and Dohme, Boehringer Ingelheim, and GlaxoSmithKline. SH declares to be engaged and paid as an expert in some scientific boards, clinical research, and lectures, and to receive invitations as a participant in different international meetings (with support fees) by Astra Zeneca, Boehringer Ingelheim, Bristol-Myers Squibb, Eli Lilly, Merck Sharp and Dohme, Novartis, Novo Nordisk, Roche Diagnostics, and Sanofi. CM has received fees for participation in an advisory board for Novo Nordisk. SP has received fees from Novo Nordisk for participation in symposia. GR has received honoraria for giving lectures in symposia organized by Abbott, Astra Zeneka, Bayer Diagnostics, Dexcom, Janssen, Lifescan, Eli Lilly, Menarini, MerckSerono, MSD, Novartis, Novo Nordisk, Roche Diagnostics, and Sanofi-Aventis; he has served on the advisory boards of Abbott, BayerDiagnostics, Lifescan, Roche Diagnostics, and Sanofi-Aventis; he has also received a grant from Lifescan for evaluating an educational tool used in functional insulin therapy; he has received honoraria from Novo Nordisk for participating in meetings analyzing the DAWN2 ${ }^{\mathrm{TM}}$ data. CTT has participated in an advisory board for Novo Nordisk concerning results of the DAWN2 ${ }^{\mathrm{TM}}$ study.

\section{References}

1. Peyrot M, Rubin RR, Lauritzen T, Snoek FJ, Matthews DR, Skovlund SE. Psychosocial problems and barriers to improved diabetes management: results of the Cross-National Diabetes Attitudes, Wishes and Needs (DAWN) Study. Diabet Med. 2005;22(10):1379-1385.

2. Rubin RR, Peyrot M, Siminerio LM. Health care and patient-reported outcomes: results of the cross-national Diabetes Attitudes, Wishes and Needs (DAWN) study. Diabetes Care. 2006;29(6):1249-1255.

3. International Diabetes Federation Clinical Guidelines Task Force. Global guideline for Type 2 diabetes. Brussels: International Diabetes Federation, 2005. Available from: http://www.idf.org/webdata/docs/IDF\%20 GGT2D.pdf. Accessed November 11, 2014.

4. Engel GL. The need for a new medical model: a challenge for biomedicine. Science. 1977;196(4286):129-136.
5. Inzucchi SE, Bergenstal RM, Buse JB, et al. Management of hyperglycemia in type 2 diabetes: a patient-centered approach: position statement of the American Diabetes Association (ADA) and the European Association for the Study of Diabetes (EASD). Diabetes Care. 2012; 35(6):1364-1379.

6. Haute Autorié de la Santé. Recommandation de bonne pratique. Stratégie médicamenteuse de contrôle glycémique du diabète de type 2. 2013 [Practice Guidelines. Therapeutic Strategy for type 2 diabetes control, 2013]. Available from: http://www.has-sante.fr/portail/upload/docs/ application/pdf/2013-02/10irp04_reco_diabete_type_2.pdf. Accessed November 11, 2014.

7. Peyrot M, Burns KK, Davies M, et al. Diabetes Attitudes Wishes and Needs 2 (DAWN2): A multinational, multi-stakeholder study of psychosocial issues in diabetes and person-centred diabetes care. Diabetes Res Clin Pract. 2013;99(2):174-184.

8. Development of the World Health Organization WHOQOL-BREF quality of life assessment. The WHOQOL Group. Psychol Med. 1998; 28(3):551-558.

9. Szende A, Oppe M, Devlin NJ, EuroQol Group. EQ-5D value sets: inventory, comparative review, and user guide. Dordrecht: Springer; 2007. ISBN: 978-1-4020-5510-2 (Print) 978-1-4020-5511-9 (Online).

10. de Wit M, Pouwer F, Gemke RJ, et al. Validation of the WHO-5 well-being index in adolescents with type 1 diabetes. Diabetes Care. 2077;30(8):2003-2006.

11. McGuire BE, Morrison TG, Hermanns N, et al. Short-form measures of diabetes-related emotional distress: the Problem Areas in Diabetes Scale (PAID)-5 and PAID-1. Diabetologia. 2010;53(1):66-69.

12. Welch G, Weinger K, Anderson B, Polonsky WH. Responsiveness of the Problem Areas In Diabetes (PAID) questionnaire. Diabet Med. 2003;20(1):69-72.

13. Parkin T, Skinner TC. Discrepancies between patient and professionals recall and perception of an outpatient consultation. Diabet Med. 2003; 20(11):909-914.

14. Toobert DJ, Hampson SE, Glasgow RE. The summary of diabetes self-care activities measure: results from 7 studies and a revised scale. Diabetes Care. 2000;23(7):943-950.

15. Anderson RM, Fitzgerald JT, Gruppen LD, Funnell MM, Oh MS. The Diabetes Empowerment Scale-Short Form (DES-SF). Diabetes Care. 2003;26(5):1641-1642.

16. Holt RI, Nicolucci A, Kovacs Burns K, et al. Diabetes Attitudes, Wishes and Needs second study (DAWN2 ${ }^{\mathrm{TM}}$ ): cross-national comparisons on barriers and resources for optimal care - healthcare professional perspective. Diabet Med. 2013;30(7):789-798.

17. Glasgow RE, Wagner EH, Schaefer J, Mahoney LD, Reid RJ, Greene SM. Development and validation of the Patient Assessment of Chronic Illness Care (PACIC). Med Care. 2005;43(5):436-444.

18. Glasgow RE, Whitesides H, Nelson CC, King DK. Use of the Patient Assessment of Chronic Illness Care (PACIC) with diabetic patients: relationship to patient characteristics, receipt of care, and self-management. Diabetes Care. 2005;28(11):2655-2661.

19. Schafer LC, McCaul KD, Glasgow RE. Supportive and nonsupportive family behaviors: relationships to adherence and metabolic control in persons with type I diabetes. Diabetes Care. 1986;9(2): 179-185.

20. Kovacs Burns K, Nicolucci A, Holt RI, et al. Diabetes Attitudes, Wishes and Needs second study (DAWN2 ${ }^{\mathrm{TM}}$ ): cross-national benchmarking indicators for family members living with people with diabetes. Diabet Med. 2013;30(7):778-788.

21. Nicolucci A, Kovacs Burns K, Holt RI, et al. Diabetes Attitudes, Wishes and Needs second study (DAWN2): cross-national benchmarking of diabetes-related psychosocial outcomes for people with diabetes. Diabet Med. 2013;30(7):767-777.

22. Hochberg G, Pucheu S, Kleinebreil L, Halimi S, Fructuoso-Voisin C. WHO-5, a tool focusing on psychological needs in patients with diabetes: the French contribution to the DAWN study. Diabetes Metab. 2012;38(6):515-522. 
23. Solli O, Stavem K, Kristiansen IS. Health-related quality of life in diabetes: The associations of complications with EQ-5D scores. Health Qual Life Outcomes. 2010;8:18.

24. Trief PM, Wade MJ, Pine D, Weinstock RS. A comparison of healthrelated quality of life of elderly and younger insulin-treated adults with diabetes. Age Ageing. 2003;32(6):613-618.

25. Anderson RJ, Freedland KE, Clouse RE, Lustman PJ. The prevalence of comorbid depression in adults with diabetes: a meta-analysis. Diabetes Care. 2001;24(6):1069-1078.

26. Phillips LS, Branch WT, Cook CB, et al. Clinical inertia. Ann Intern Med. 2001;135(9):825-834.

27. Lustman PJ, Anderson RJ, Freedland KE, de Groot M, Carney RM, Clouse RE. Depression and poor glycemic control: a meta-analytic review of the literature. Diabetes Care. 2000;23(7):934-942.

28. de Groot M, Anderson R, Freedland KE, Clouse RE, Lustman PJ. Association of depression and diabetes complications: a meta-analysis. Psychosom Med. 2001;63(4):619-630.

29. Lin EH, Heckbert SR, Rutter CM, et al. Depression and increased mortality in diabetes: unexpected causes of death. Ann Fam Med. 2009; 7(5):414-421.
30. Barnard K, Thomas S, Royle P, Noyes K, Waugh N. Fear of hypoglycaemia in parents of young children with type 1 diabetes: a systematic review. BMC Pediatr. 2010;10:50.

31. White P, Smith SM, O’Dowd T. Living with Type 2 diabetes: a family perspective. Diabet Med. 2007;24(7):796-801.

32. Peyrot M, Rubin RR, Lauritzen T, et al. Resistance to insulin therapy among patients and providers: results of the cross-national Diabetes Attitudes, Wishes, and Needs (DAWN) study. Diabetes Care. 2005; 28(11):2673-2679.

33. Polonsky WH, Fisher L, Guzman S, Villa-Caballero L, Edelman SV. Psychological insulin resistance in patients with type 2 diabetes: the scope of the problem. Diabetes Care. 2005;28(10):2543-2545.

34. Clark M, Hampson SE. Comparison of patients' and healthcare professionals' beliefs about and attitudes towards Type 2 diabetes. Diabet Med. 2003;20(2):152-154.

35. Schillinger D, Piette J, Grumbach K, et al. Closing the loop: physician communication with diabetic patients who have low health literacy. Arch Intern Med. 2003;163(1):83-90.
Patient Preference and Adherence

\section{Publish your work in this journal}

Patient Preference and Adherence is an international, peer-reviewed, open access journal that focuses on the growing importance of patient preference and adherence throughout the therapeutic continuum. Patient satisfaction, acceptability, quality of life, compliance, persistence and their role in developing new therapeutic modalities and compounds to optimize

\section{Dovepress}

clinical outcomes for existing disease states are major areas of interest for the journal. This journal has been accepted for indexing on PubMed Central. The manuscript management system is completely online and includes a very quick and fair peer-review system, which is all easy to use. Visit http://www. dovepress.com/testimonials.php to read real quotes from published authors. 